(RESEARCH ARTICLE)

\title{
A new investigation on MHD Stoke's flow with induced magnetic field effect
}

\author{
Ghosh Swapan Kumar* \\ Department of Mathematics, Narajole Raj College, Narajole, Dist- Paschim Midnapore - 721211 West Bengal, India.
}

Publication history: Received on 13 December 2019; revised on 26 December 2019; accepted on 27 December 2019

Article DOI: https://doi.org/10.30574/gjeta.2019.1.1.0011

\begin{abstract}
Unsteady MHD Stoke's flow of a viscous incompressible electrically conducting fluid past a flat plate under the influence of a transverse magnetic field is studied. The induced magnetic field produced by the motion of an electrically conducting fluid which perturb the original magnetic field. Since the plate is considered perfectly conducting, the magnetic field does not penetrate outside the region space. This problem is solved by employing Laplace transform method. The effect of induced magnetic field plays an important role in determining the MHD Stoke's flow behaviour. Numerical results of velocity distributions, induced magnetic field distributions and the shear stress at the plate are depicted graphically for various values of flow parameters. This gives a physical interpretation obtained by the numerical results.
\end{abstract}

Keywords: Unsteady flow; MHD Stoke's flow; Induced magnetic field; Laplace transform method; Perfectly conducting plate

\section{Nomenclature}

q - Velocity vector; U - Velocity of the plate; B - Magnetic field vector; $t^{\prime}$ - Time; E - Electric field vector; $B_{0}$ - Magnetic flux density; J - Current density vector; $\mathrm{M}$ - Hartmann number; $\mathrm{H}$ - Intensity of a magnetic field vector; $P_{m}$ - Magnetic Prandtl number; $u^{\prime}$ - Velocity component; $y^{\prime}$ - Distance; $B_{x}^{\prime}$ - Magnetic field component; $\tau_{x}$ - Shear stress.

\section{Greek symbols}

$\rho$ - Fluid density; $\sigma$ - Electrical conductivity; $\mu_{e}$ - Magnetic permeability; $\eta$ - Magnetic diffusivity; $v$ - Kinematic coefficient of viscosity

\section{Introduction}

Magnetohydrodynamic (MHD) Stoke's flow has received a challenging approach to researchers with a view to analyze the area of plasma dynamics by mathematical method. MHD Stoke's flow continues its scientific approach to determine the flow behaviour by applying mathematical method with different conditions and configurations. However, it finds a considerable attention to many researchers leading to a MHD channel flow by applying analytical method to exerts its influence of induced magnetic field effect. This has been studied by Gupta[1], Denno and Fouad[2], Mazumder et.al[3], Ghosh[4-5], Ghosh and Nandi[6], Ghosh and Bhattacharjee[7], Ghosh[8], Ghosh et.al[9], Beg et.al[10] and Ghosh et.al[11]. MHD flow past a flat plate by considering the effect of induced magnetic field has been studied by Ghosh and Pop [12] and Ghosh et al. [13]. Although radiative heat transfer aspect on hydrodynamic flow past a flat plate has been considered by many authors by employing Laplace transform method, neglecting induced magnetic field effect. Mention may be made of their works of Fujii and Imura [14], Lewandowski [15], Raptis and Perdikis[16], Ghosh and Pop[17], Ghosh[18] and Beg et.al[19]. Magnetohydrodynamic flow with radiative heat transfer past a flat plate has been studied by their works of Helmy[20] and Ghosh et.al[21] with reference to Laplace transform method where the effect of

\footnotetext{
* Corresponding author

E-mail address: g_swapan2002@yahoo.com
} 
induced magnetic field becomes ignored. A literature survey reveals that none of the researcher has been found to work with the effect of induced magnetic field on MHD flow regime by applying Laplace transform method. The aim of present investigation is to deal with the study of a transient MHD Stoke's flow in the presence of a transverse magnetic field where electric field cannot be ignored. Therefore, the effect of induced magnetic field has a pronounced effect on the MHD flow regime. The effect of induced magnetic field becomes significant to the flow region pose formidable mathematical challenge by applying Laplace transform method. This investigation leads to a fundamental behaviour on MHD Stoke's flow which has not received attention in literature. Such type of flow behaviour in the presence of magnetic field is subjected to a non-catalytic system in such a way that the homogeneous reaction takes place of equal number of ions and electrons. In turn, the behaviour of a charged particle under the influence of a strong magnetic field immense a scientific occurrence of a plasma state inside the MHD flow region. The effect of induced magnetic field inside the MHD flow regime perturbs the original fluid motion in a perfectly conducting plate. This situation reveals that the magnetic field does not penetrate outside the flow region. The flow of electron inside the region of space has become a greater importance to radio wave propagation to exert its influence of a microwave background of radiation. Indeed, plasma behaviour inside the MHD flow region lies in its application in construction of nuclear reactor, solar power generator and space craft propulsion system.

The purpose of present investigation leads to the study of an unsteady magnetohydrodynamicStoke's flow of a viscous incompressible electrically conducting fluid occupied a semi-infinite region of space bounded by an infinite flat plate in the presence of transverse magnetic field. This problem is treated as MHD startup flow. The MHD flow close to plate is suddenly set into motion with a constant velocity in its own plane. The effect of induced magnetic field becomes significant with reference to $\nabla \times E=-\frac{\partial B}{\partial t}$ with a decisive importance to a magnetic field and Maxwell displacement current. The influence of an MHD Stoke's flow in a perfectly conducting plate takes place of an induced magnetic field which perturb the original magnetic field inside the region of space. In a realistic situation, since the MHD flow is generated by the equal number of ions and electrons in the presence of strong magnetic field, the plasma behaviour inside the flow regime with high electrical conductivity implies to a controllable region in a nuclear reactor with the representation of a tokamak. Representing the analysis of an MHD Stoke's flow in the presence of a transverse magnetic field, the electric field cannotbe ignored so that the effect of induced magnetic field has a pronounced effect on the MHD flow region.

\section{Formulation of the problem and its solutions}

Consider an unsteady flow of a viscous incompressible electrically conducting fluid occupying a semi-infinite region of space bounded by an infinite flat plate. An uniform transverse magnetic field is applied perpendicular to the plane. This problem is treated as startup flow. The flow close to the plate is suddenly set into motion with a constant velocity $\mathrm{U}$ in its own plane. Since the plate lies along $\mathrm{x}^{\prime}-$ axis, the coordinate system reveals to $\mathrm{x}^{\prime}-$ axis along the plate and $\mathrm{y}^{\prime}-$ axis is normal to it. Since pressure is constant in the whole space, an infinity extended plate along $\mathrm{x}^{\prime}-$ axis it reveals that all physical quantities except pressure will be function of $\mathrm{y}^{\prime}$ and $\mathrm{t}^{\prime}$ only.

The MHD equation of motion reads

$\frac{\partial q}{\partial t}=v \nabla^{2} q+\frac{1}{\rho \mu_{e}}(\nabla \times \mathrm{B}) \times B$

The conservation of mass leads to the equation of continuity

$\nabla \cdot \mathrm{q}=0$

The solenoidal relation that the magnetic field produces no source or sink reads

$\nabla \cdot \mathrm{B}=0$

Since the motion becomes unsteady, the Maxwell equation becomes

$\nabla \times \mathrm{E}=-\frac{\partial B}{\partial t}$

The Ampere's law becomes

$\nabla \times \mathrm{B}=\mu_{e} J$ 
The other Maxwell equations are

$\nabla \times \mathrm{H}=\mathrm{J}$

The conservation of electric charge reads

$\nabla . J=0$

Where $\mathrm{B}=\mu_{e} H$

The Ohm's law for a moving conductor

$\mathrm{J}=\sigma[E+q \times B]$

Equation(8) is closely resemblance to the magnetic induction equation which leads to

$\frac{\partial B}{\partial t}=\operatorname{curl}(q \times B)+\eta \nabla^{2} B$

Where $\eta=\frac{1}{\sigma \mu_{e}}$ is the magnetic diffusivity or viscosity. q,B,E and J are, respectively, the velocity vector, magnetic field vector, electric field vector and current density vector. Further, $\rho, \mu_{e}, v, \sigma$ and $\mathrm{t}$ are the fluid density, magnetic permeability, kinematic coefficient of viscosity, electrical conductivity and time.

According to geometry of the problem, equations(1) to (9) are in agreement with the following equations reads

The MHD equation of motion

$\frac{\partial u^{\prime}}{\partial t^{\prime}}=v \frac{\partial^{2} u^{\prime}}{\partial y^{\prime 2}}+\frac{1}{\mu_{e} \rho}\left(\nabla \times B^{\prime}\right) \times B^{\prime}(10)$

The magnetic induction equation becomes

$\frac{\partial B_{x}^{\prime}}{\partial t^{\prime}}=\operatorname{curl}\left(u^{\prime} \times B_{x}^{\prime}\right)+\frac{1}{\sigma \mu_{e}} \nabla^{2} B_{x}^{\prime}$

The initial and the boundary conditions for velocity become

$u^{\prime}=0$ for all $y^{\prime} \geq 0$ and $t^{\prime} \leq 0$,

$u^{\prime}=U$ at $y^{\prime}=0$ for $t^{\prime}>0$,

$$
u^{\prime} \rightarrow 0 \text { as } y^{\prime} \rightarrow \infty \text { for } t^{\prime}>0
$$

Since the plate is considered perfectly conducting, the following magnetic boundary conditions are

$\frac{d B_{x}^{\prime}}{d y^{\prime}}=0 \mathrm{at} y^{\prime}=0$

$\frac{d B_{x}^{\prime}}{d y^{\prime}}=0$ at $y^{\prime} \rightarrow \infty$

$B_{x}^{\prime} \rightarrow 0$ asy $y^{\prime} \rightarrow \infty$

Introducing dimensionless quantities

$\mathrm{u}=\frac{u^{\prime}}{U}, \quad \tau=\frac{t^{\prime} U^{2}}{v}, \mathrm{y}=\frac{y^{\prime} U}{v}, B_{x}=\frac{B_{x}^{\prime}}{\sigma \mu_{e} v B_{0}}$ and $M^{2}=\frac{\sigma B_{0}^{2} v}{\rho U^{2}}$ is the square of Hartmann number where $B_{0}$ is the magnetic flux density.

Equations (10) and (11) can be transformed into a dimensionless form read 
$\frac{\partial u}{\partial \tau}=\frac{\partial^{2} u}{\partial y^{2}}+M^{2} \frac{\partial B_{x}}{\partial y}$

$P_{m} \frac{\partial B_{x}}{\partial \tau}=\frac{\partial u}{\partial y}+\frac{\partial^{2} B_{x}}{\partial y^{2}}$

Where $P_{m}=\sigma \mu_{e} v$ is the magnetic Prandtl number.

The corresponding velocity boundary conditions are

$\mathrm{u}=0$ for all $y \geq 0$ and $\tau \leq 0$,

$\mathrm{u}=1$ at $y=0$ for $\tau>0$,

$u \rightarrow 0$ as $y \rightarrow \infty$ for $\tau>0$

and the corresponding magnetic boundary conditions become

$$
\frac{d B_{x}}{d y}=0 \quad \text { at } y=0
$$

$\frac{d B_{x}}{d y} \rightarrow 0 \quad$ as $y \rightarrow \infty$

$$
B_{x} \rightarrow 0 \quad \text { as } y \rightarrow \infty
$$

With reference to Laplace transform method, the following equations (15) and (16) subject to boundary conditions (17) and (18) take the form

$s u^{*}=\frac{\partial^{2} u^{*}}{\partial y^{2}}+M^{2} \frac{\partial B_{x}^{*}}{\partial y}$

$P_{m} s B_{x}^{*}=\frac{\partial^{2} B_{x}^{*}}{\partial y^{2}}+\frac{\partial u^{*}}{\partial y}$

The corresponding velocity boundary conditions are

$$
u^{*}=0 \quad \text { for all } y \geq 0 \text { and } \tau \leq 0,
$$

$u^{*}=\frac{1}{s} \quad$ at $y=0$ for $\tau>0$,

$$
u^{*} \rightarrow 0 \quad \text { as } y \rightarrow \infty \text { for } \tau>0
$$

The corresponding magnetic boundary conditions are

$$
\frac{d B_{x}^{*}}{d y}=0 \quad \text { at } y=0,
$$

$\frac{d B_{x}^{*}}{d y} \rightarrow 0 \quad$ at $y \rightarrow \infty$,

$$
B_{x}^{*} \rightarrow 0 \quad \text { at } y \rightarrow \infty
$$

To analyze equation (20), the following equation takes the form

$\frac{d B_{\chi}^{*}}{d y}=P_{m} s\left[B_{x}^{*} y-\int y \frac{d B_{\chi}^{*}}{d y} d y\right]-u^{*}+C_{1}$

Where $C_{1}$ is arbitrary constant. 
Proceeding in this way, equations (19) to (23) subject to boundary conditions (21) and (22) the solutions of velocity distribution and induced magnetic field distributions can be obtained by employing inverse Laplace transform method such as

Velocity distribution $\mathrm{u}(\mathrm{y}, \tau)$

$u(y, \tau)=e^{-M y} \cdot \operatorname{erfc}\left(\frac{y}{2 \sqrt{\tau}}-M \sqrt{\tau}\right)+e^{M y} \cdot \operatorname{erfc}\left(\frac{y}{2 \sqrt{\tau}}+M \sqrt{\tau}\right)-e^{-M^{2} \tau} \cdot \operatorname{erfc}\left(\frac{y}{2 \sqrt{\tau}}\right)-\frac{1}{2}\left[e^{-2 M y} \cdot \operatorname{erfc}\left(\frac{y}{\sqrt{\tau}}-M \sqrt{\tau}\right)+\right.$
$\left.e^{2 M y} \cdot \operatorname{erfc}\left(\frac{y}{\sqrt{\tau}}+M \sqrt{\tau}\right)\right]+e^{-M^{2} \tau} \cdot \operatorname{erfc}\left(\frac{y}{\sqrt{\tau}}\right)$

Induced magnetic field distribution $B_{x}(y, \tau)$

$B_{x}(y, \tau)=y e^{-M^{2} \tau} \cdot \operatorname{erfc}\left(\frac{y}{2 \sqrt{\tau}}\right)-2 \sqrt{\frac{\tau}{\pi}} e^{-\left(M^{2} \tau+\frac{y^{2}}{4 \tau}\right)}-y e^{-M^{2 \tau}} \cdot \operatorname{erfc}\left(\frac{y}{\sqrt{\tau}}\right)+\sqrt{\frac{\tau}{\pi}} e^{-\left(M^{2} \tau+\frac{y^{2}}{\tau}\right)}$

Frictional shearing stress at the plate $\mathrm{y}=0$ becomes

$\tau_{x}=\left(\frac{d u}{d y}\right)_{y=0}=-\frac{1}{\sqrt{\pi \tau}} e^{-M^{2} \tau}$

\section{Results and discussion}

A physical interpretation demonstrates the MHD Stoke's flow behaviour in consonance with numerical results implying thereby that leads to the graphical representations for various values of $M$ (magnetic force) and $\tau$ (time) in Figures 1 to 5. Following the analysis of velocity distribution, induced magnetic field distribution and the frictional shearing stress; the mathematical method exerted by Laplace transform method which implicates the solution of induced magnetic field distribution and does over the flow situation where the effects of induced magnetic field has not been taken into account since 1942. A literary survey reveals to an MHD Stoke's flow with different conditions and configurations by neglecting induced magnetic field effects under the implication of Laplace transform method. The aim of a physical discussion exerts its influence of ionized flow with a perfectly conducting plate with a view to analyze MHD Stoke's flow behaviour.

Figures 1 and 2 for velocity distributions take place in a skewed nature that converges near the plate. Since the profiles are skewed in nature, the skewness is characterized by the strong magnetic pressure due to the presence of a Lorentz force. Fig. 1 shows that the flow velocity behaves in a manner trusted to fall into the region of space due to the presence of Lorentz force on increasing the M (the magnetic pressure) for fixed value of $\tau$ (time).

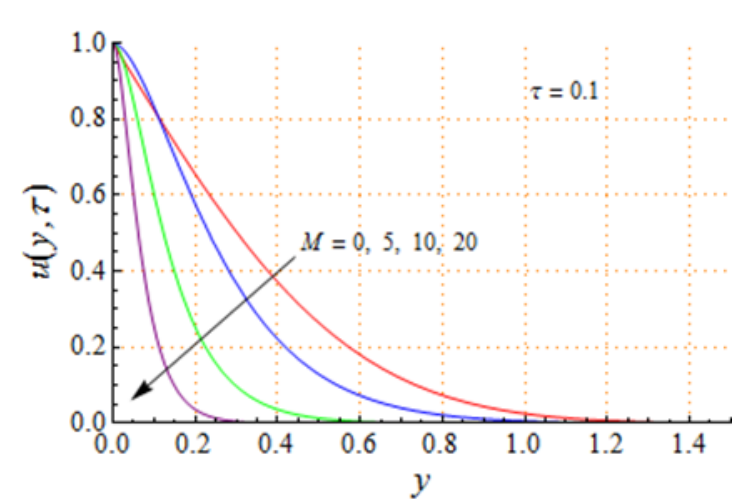

Figure 1 Velocity profile on varying M

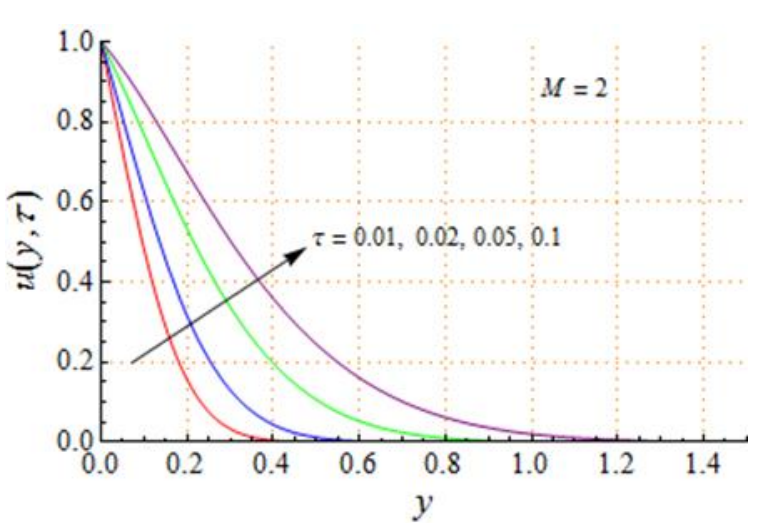

Figure 2 Velocity profile on varying time $\tau$ 


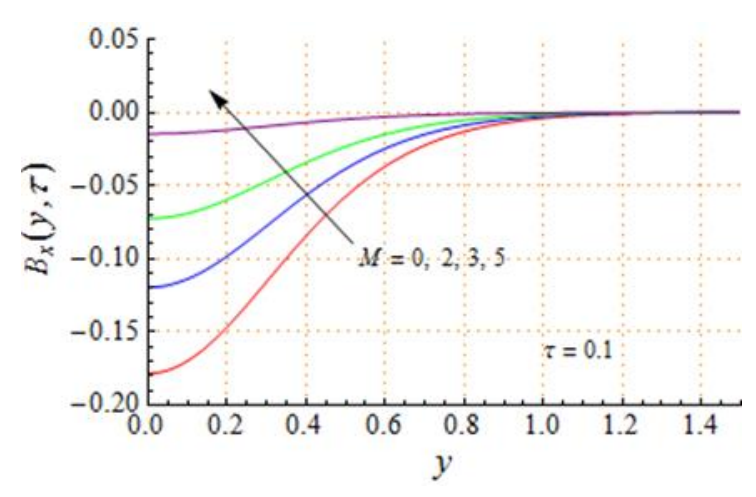

Figure 3 Induced magnetic field profile on varying M

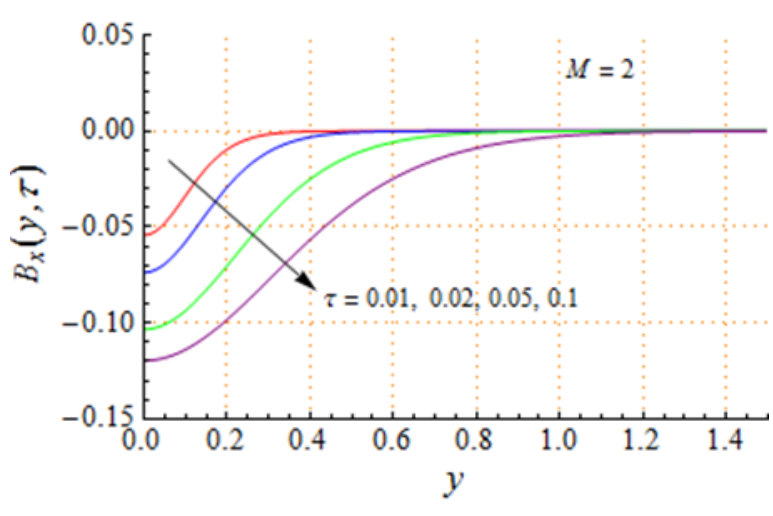

Figure 4 Induced magnetic field profile on varying time $\tau$

The flow velocity into the region on space is compatible with equal number of ions and electrons subjected to an ionized flow so that magnetic force plays an important role in an electromagnetic field. In this situation, an ionized plasma flow with high electrical conductivity in a controllable region of space through the perfectly conducting plate that the magnetic field does not penetrate outside the region of space. In such a case, the velocity of plasma flow has become a tendency to fall into the region and it has no effect far away from the plate so that all the velocity profiles become quickly converge near the plate. Fig. 2 reveals that the flow velocity increases with the increase in time $(\tau)$. It happens in the case of a velocity field that time accelerates the MHD flow field to boost up Lorentz force. A time oriented velocity field depends on magnetic force to accelerate the MHD flow situation with the increase in time $(\tau)$ caused by the magnetic pumping and the velocity dies out quickly near the plate. It is evident from Fig. 3 that the induced magnetic field increases with the increase in M (magnetic force). The effect of induced magnetic field becomes reverse from the plate which can be entrusted by the reversal induced magnetic field as the induced magnetic field produced by the motion of an electrically conducting fluid which perturb the original magnetic field. Since the magnetic force varies with time, the reverse induced magnetic field occurs due to the presence of a magnetic pumping. In this situation, the magnetic lines of force is to be forced outward from the plate in a region of space so that the magnetic lines of force cuts from the fluid region and it converges quickly near the place. In a controllable region of space, the plasma flow behaves with equal number of ions and electrons in the presence of a strong magnetic field so that the effect of induced magnetic field plays an important role in a fusion reactor. In turn, the induced magnetic field gyrates round the lines of force to accelerate the flow situation. Indeed, the induced magnetic field produced by the motion of an electrically conducting fluid becomes significant where electric field cannot be ignored. Fig. 4 demonstrates that a time $(\tau)$ variation with a fixed value of $M$ (magnetic force) leads to fall the effect of induced magnetic field to exert its influence on reversal of an induced magnetic field near the plate and it converges quickly near the plate. The skewness of all the profiles immense a satisfactory explanation with reference to a magnetic pressure on the effect of induced magnetic field. The reversal of an induced magnetic field with a time variation corresponds to a impulsive onset into the motion. Although, a time variation with magnetic force represents magnetic pumping to exhibit a time elapse far away from the plate.

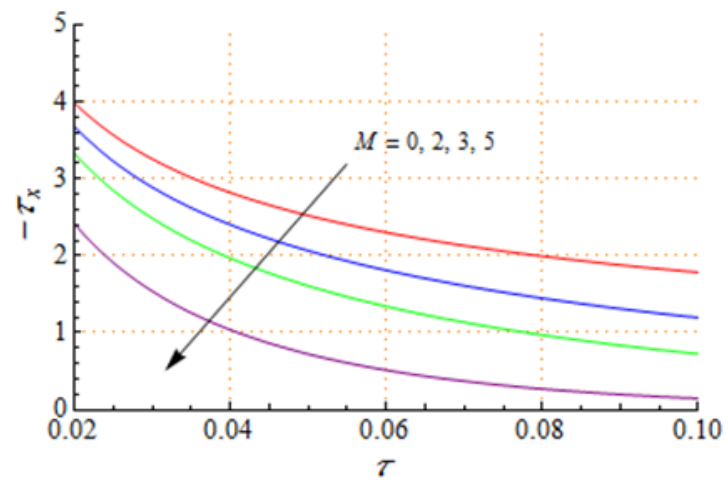

Figure 5 Variation of shear stress on varying $M$ and time $\tau$

It is noticed from Fig. 5 that the frictional shearing stress at the plate reveals to the variation of a magnetic force (M) and the time $(\tau)$. The frictional shearing stress $\left(\tau_{x}\right)$ decreases at the plate with an increase of a magnetic force (M) while 
the frictional shearing stress $\left(\tau_{x}\right)$ increases in magnitude with the variation of time $(\tau)$ for a fixed value of a magnetic force (M) and then it decreases in magnitude with the increase in time $(\tau)$. Here, separation does not exist so that no back flow occurs. Since frictional shear stress $\left(\tau_{x}\right)$ depends on a magnetic force $(\mathrm{M})$, the viscous force offers a resistance to its motion which is balanced by a magnetic force (M). Therefore, the influence of a magnetic force on frictional shearing stress $\left(\tau_{x}\right)$ tends to stabilize the MHD flow situation in a region of space. Finally, the frictional shear stress $\left(\tau_{x}\right)$ restore the medium in a controllable region under the influence of a magnetic force.

\section{Conclusion}

Transient MHD Stoke's flow of a viscous incompressible electrically conducting fluid past a flat plate in the presence of a transverse magnetic field is investigated. The induced magnetic field produced by the motion of an electrically conducting fluid resulting from a transverse magnetic field that cannot be ignored due to the presence of an electric field and Maxwell's displacement current. Therefore, the effect of induced magnetic field becomes predominant over the entire flow regime. The plate is considered perfectly conducting so that the magnetic field does not penetrate outside the flow region. Since an MHD Stoke's flow is entrusted by the equal number of ions and electrons in a homogenous medium, the plasma flow inside the flow regime with high electrical conductivity implies to a controllable region of space in the electromagnetic field where electric field sustained the flow regime.

\section{Compliance with ethical standards}

\section{Acknowledgments}

Author wishes to express his sincere thanks to Narajole Raj College for his research work.

\section{Disclosure of conflict of interest}

Author claims that he has no conflict of interest.

\section{References}

[1] Gupta AS. (1969). Combined free and forced convection effects on the magnetohydrodynamic flow through a channel. ZAMM, 20, 516 - 512.

[2] Denno KI and Fouad AA. (1972). Effect of induced magnetic field on magnetohydrodynamic channel flow, IEEE Trans Electron Dev, 19, 322 - 331.

[3] Mazumder BS, Gupta AS and Datta N. (1976). Hall effects on combined free and forced convective hydromagnetic flow through a channel. Int. J Eng Sci, 14, 285 - 292.

[4] Ghosh SK. (1991). A note on steady and unsteady hydromagnetic flow in a rotating channel in the presence of inclined magnetic field. Int J Eng Sci, 29, 1013 - 1016.

[5] Ghosh SK. (1993). Unsteady hydromagnetic flow in a rotating channel with oscillating pressure gradient. J Phys Soc Jpn, 62, $3893-3903$

[6] Ghosh SK and Nandi DK. (2000). Magnetohydrodynamic fully developed combined convection flow between vertical plates heated asymmetrically. J Tech Phys, 41, 173 - 185

[7] Ghosh SK and Bhattacharjee PK. (2000). Magnetohydrodynamic convective flow Archives of Mechanics, 52, 303 -318 .

[8] Ghosh SK. (2002). Effects of Hall current on MHD Couette flow in a rotating system with arbitrary magnetic field. Czech J Phys, 52, $51-63$.

[9] Ghosh SK, Anwar Beg O and Narahari M. (2009). Hall effects on MHD flow in a rotating system with heat transfer characteristics. Meccanica, 44, $741-765$.

[10] Anwar Beg O, Bakier AY, Prasad VR, Zueco J and Ghosh SK. (2009). Non-similar, laminar, steady, electrically conducting forced convection liquid metal boundary layer flow with induced magnetic field effects. Int. J. Thermal Sci, 48, $1596-1606$. 
[11] Ghosh SK, Beg OA, Zueco J and Prasad VR. (2010). Transient hydromagnetic flow in a rotating channel permeated by an inclined magnetic field with magnetic induction and Mazwell displacement current effects. Z Angew Math Phys (ZAMP), 61, 147 - 169.

[12] Ghosh SK and Pop I. (2006). A new approach on MHD natural convection boundary layer flow past a flat plate of finite dimension. J Heat Mass Transfer, 42, 587 - 595.

[13] Ghosh SK, Anwar Beg $O$ and Zueco J. (2010). Hydromagnetic free convection flow with induced magnetic field effects. Meccanica, 45, 175 - 185.

[14] Fujii T and Imura H. (1972). Natural convection heat transfer from a plate with arbitrary inclination. Int. J Heat Mass Transfer, 15, $755-764$.

[15] Lewandowski WM. (1991). Natural convection heat transfer from plates of finite dimension. Int. J Heat Mass Transfer, 34, 875 - 885.

[16] Raptis A and Perdikis C. (2003). Thermal radiation of an optically thin gray gas. Int J ApplMechEng, 8, 131 - 134.

[17] Ghosh SK and Pop I. (2007). Thermal radiation of an optically thick gray gas in the presence of indirect natural convection. Int J Fluid Mech Res, 34, 515 - 520.

[18] Ghosh SK. (2007). Thermal radiation of optically thick gray gas in the presence of indirect natural convection. Int J ApplMechEng, 12, 593 - 598.

[19] Anwar Beg 0, Ghosh SK, Narahari M and Beg Tasveer A. (2011). Mathematical modeling of thermal radiation effects on transient gravity-driven optically thick gray convection flow along an inclined plane with pressure gradient. ChemEngCommun, 198, 1630 - 1644.

[20] Hemly AK. (1999). MHD unsteady free convection flow past a vertical porous plate. Z Angew Math Mech (ZAMM), $78,225-270$.

[21] Ghosh SK, Das S and Jana RN. (2015). Transient MHD free convective flow of an optically thick gray gas past a moving vertical plate in the presence of thermal radiation and mass diffusion. J Appl Fluid Mech, 8, 65 - 73.

\section{How to cite this article}

Ghosh SK. (2019). A new investigation on MHD Stoke's flow with induced magnetic field effect. Global Journal of Engineering and Technology Advances, 1(1), 49-56. 